\title{
Estratégia Teórico-Prática no Ensino de Enfermagem em Doenças Transmissíveis com o Foco na Vigilância em Saúde
}

\author{
Érica Gomes Pereira ${ }^{1 *}$, Sara de Alencar Ciaccio ${ }^{2}$, Lucia Yasuko Izumi Nichiata ${ }^{1}$, Karen Namie \\ Sakata-So', João Henrique de Morais Ribeiro' ${ }^{1}$, Caroline Lopes Ciofi Silva ${ }^{1}$, Maria Clara \\ Padoveze ${ }^{1}$
}

${ }^{1}$ Departamento de Enfermagem em Saúde Coletiva da Escola de Enfermagem da Universidade de São Paulo ${ }^{2}$ Unidade de Vigilância em Saúde Butantã da Secretaria Municipal de Saúde de São Paulo/SP

* Autora para correspondência: egpereira@usp.br

\section{RESUMO}

Este relato apresenta a estratégia de ensino teórico-prática para o conteúdo de vigilância em saúde na Disciplina ENS 0425 - Enfermagem em Doenças Transmissíveis com Enfoque em Saúde Coletiva, do curso de bacharelado em Enfermagem, por meio do estágio em unidades de vigilância em saúde localizadas na cidade de São Paulo no ano de 2016. As atividades em campo de estágio tiveram como resultado a produção de relatórios em formato de estudo de caso, tanto no âmbito individual quanto no âmbito populacional de enfrentamento das doenças transmissíveis. A estratégia de ensino pode ser considerada inovadora, porque estimulou o estudante ao desenvolvimento do trabalho articulado com diversos atores envolvidos na vigilância em saúde e ao debate sobre os conceitos de morbidade, letalidade, prevalência e incidência, tendo como ponto de partida a desigualdade territorial das necessidades em saúde com vistas à atuação individual e populacional.

Palavras-Chave: Bacharelado em Enfermagem; Doenças Transmissíveis; Vigilância em Saúde Pública.

\begin{abstract}
This report presents the strategy of theoretical and practical teaching for the content of health surveillance in elective course ENS 0425 - Nursing in Communicable Diseases with a Focus on Collective Health, of the baccalaureate course in Nursing, by means of the internship in localized health surveillance units in the city of São Paulo in 2016. The activities in the field of internships have resulted in the production of reports in a case study format both in the individual and in the population context of coping with communicable diseases. The teaching strategy can be considered innovative because it has stimulated the student to develop the work articulated with several actors involved in health surveillance and the debate on the concepts of morbidity, lethality, prevalence and incidence, starting from the territorial inequality of the needs in health for individual and population performance.
\end{abstract}

Keywords: Education Nursing; Communicable Diseases; Public Health Surveillance.

\section{Introdução}

O presente relato tem como objetivo apresentar a estratégia de ensino teórico-prática no ensino de Enfermagem em Doenças Transmissíveis para o desenvolvimento do conteúdo de vigilância em saúde, conduzida em 2016, por meio do estágio em unidades de vigilância em saúde localizadas na cidade de São Paulo.

A Saúde Coletiva é um campo de conhecimentos e práticas que se fundamenta na problematização das causas das doenças a partir dos processos de produção e reprodução social e, nessa perspectiva, busca-se a ampliação da formação de profissionais de saúde para além da técnica e da dimensão biológica (NUNES, 2000).

$\mathrm{O}$ conceito de vigilância foi introduzido no final do século XIX para observar os comunicantes ou um caso clínico, com o intuito de detectar rapidamente os sintomas de uma doença transmissível e instituir o isolamento e quarentena (WALDMAN, 2006).

No século XX, as transformações sucessivas no conhecimento científico em saúde 
possibilitaram a sistematização da vigilância em duas instâncias: em nível local - identifica e controla as doenças transmissíveis na área adscrita por um serviço de saúde; em nível regional - elabora, à luz do conhecimento científico e da análise das informações enviadas pelos serviços de saúde, o boletim epidemiológico das doenças transmissíveis (ARREAZA \& MORAES, 2010; OLIVEIRA \& GRUZ, 2015).

Na Escola de Enfermagem da Universidade de São Paulo (EE/USP), o conteúdo de vigilância em saúde aplicado ao controle das doenças transmissíveis é oferecido na Disciplina Enfermagem em Doenças Transmissíveis com Enfoque em Saúde Coletiva, do curso de bacharelado em Enfermagem (ENS 0425), aos estudantes do curso de bacharelado em Enfermagem no sétimo semestre, com carga horária total de 120 horas e para até oitenta estudantes/ano.

A disciplina tem como objetivo principal interpretar a ocorrência e o enfrentamento das doenças transmissíveis e desenvolver práticas de enfermagem voltadas às ações de vigilância em saúde.

Em 2016, o conteúdo programático agregou os seguintes temas: panorama das doenças transmissíveis; atualização nos calendários de vacina; assistência e vigilância em saúde aplicada ao controle das arboviroses, das infecções sexualmente transmissíveis, das hepatites virais, do HIV/Aids, das meningites, da influenza, da hanseníase, da leptospirose, da varicela, das doenças respiratórias prevalentes na infância, das doenças de transmissão hídrica e alimentar, do sarampo, da resistência microbiana, da tuberculose, da leishmaniose e da sífilis. A disciplina contou com seis professores doutores, quatro especialistas e com quatro alunos de pós-graduação em formação docente no Programa de Aperfeiçoamento em Ensino (PAE) que auxiliaram a supervisionar os campos de estágio com apoio dos profissionais de saúde dos serviços.

As atividades em campo de estágio tiveram como objetivo a integração de práticas individuais e coletivas de atuação dos estudantes frente às doenças transmissíveis. E, como resultado, a produção de relatórios em formato de estudo 120 de caso, tanto no âmbito individual quanto no âmbito populacional de enfrentamento das doenças transmissíveis.

\section{Desenvolvimento da Estratégia de Ensino}

Os 62 estudantes que cursaram a referida disciplina no primeiro semestre de 2016 foram divididos inicialmente em duas equipes, subdivididas em quatro subequipes (Figura 1). Eles vivenciaram os dois eixos (assistência e vigilância) ao longo da disciplina. Cada subequipe teve de sete a oito estudantes, com carga horária teórico-prática total de 56 horas.

O eixo Assistência (31 estudantes) foi composto por quatro serviços: dois hospitais escola públicos na região oeste da cidade de São Paulo e dois ambulatórios públicos localizados na região sul e na região central de São Paulo/SP. Neste eixo foram realizadas ações de vigilância em saúde, no âmbito do indivíduo.

O eixo Vigilância (31 estudantes) foi composto por três unidades municipais de vigilância em saúde e uma unidade estadual de vigilância em saúde com atuação concomitante no Laboratório de Habilidades de Enfermagem para simulação clínica/epidemiológica de enfrentamento das arboviroses. Foram elaborados dez boletins epidemiológicos com seu respectivo plano de ações para enfrentamento de uma doença transmissível mais recorrente num certo território (âmbito epidemiológico - atuação populacional).

Todos os estudos de caso, em âmbito populacional, deveriam responder a seguinte questão problematizadora: "Como correlacionar dados individuais e coletivos, tomando como base uma dada situação epidemiológica, o território e a rede de serviços de assistência e vigilância em saúde para a proposição de um plano epidemiológico de enfrentamento a uma certa doença transmissível?".

De acordo com os resultados das avaliações dos estudantes, observou-se que a participação nas atividades teórico-práticas possibilitou a ampliação do raciocínio clínico (individual) para o raciocínio epidemiológico (populacional), a descoberta de um campo de atuação que é pouco 


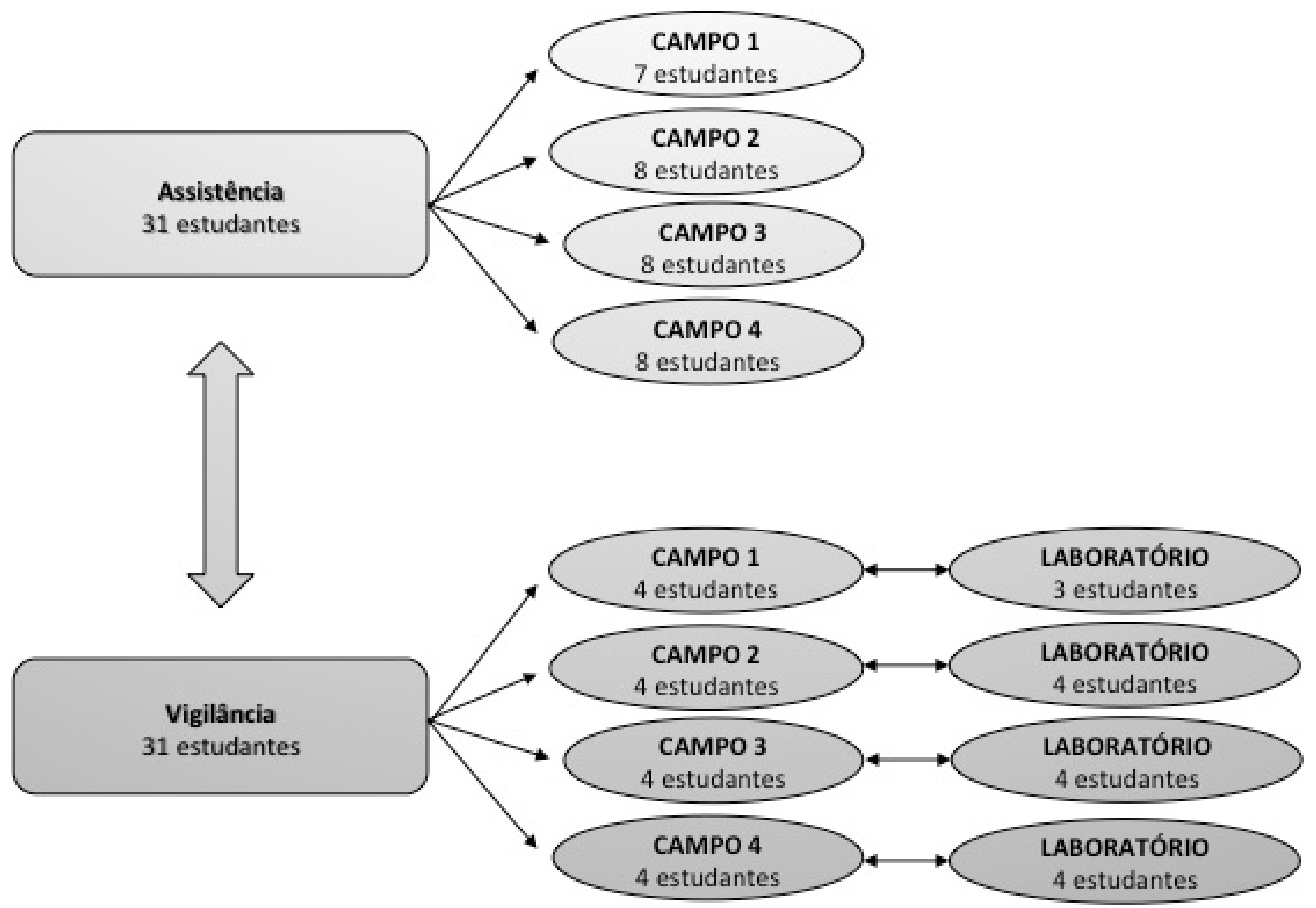

Figura 1 - Fluxograma de organização das estratégias teórico-práticas da disciplina.

abordado durante o curso de bacharelado em Enfermagem, a percepção in loco de que a intervenção populacional em saúde é efetiva quando há debate e construção coletiva com os diversos profissionais (odontólogos, enfermeiros, médicos, veterinários, entre outros) que compõem a equipe de uma unidade de vigilância em saúde.

A mediação pedagógica exercida pelos supervisores de estágio, com o apoio dos profissionais das unidades de vigilância em saúde, contribuiu para a ampliação do processo formativo com vistas a atuar em saúde e em Enfermagem. A experiência de ensinar o processo de construção de um plano de ações de alcance populacional para enfrentamento de uma dada doença transmissível implicou a retomada de conteúdos de sociologia, microbiologia, biossegurança, imunização, estatística, epidemiologia, entre outros que foram tratados do primeiro até o sexto semestre do curso, e o reconhecimento de que a história natural das doenças de notificação compulsória num certo território é determinada pela história social das desigualdades em saúde deste mesmo território.

\section{Considerações Finais}

A estratégia de ensino empreendida pela disciplina possibilitou a reflexão e a proposição de ações para além da dimensão biológica individual. Também apoiou a compreensão de que a condição de saúde de uma certa população está diretamente relacionada ao enfrentamento coletivo dos potenciais de fortalecimento e desgaste no trabalho, acesso à moradia, à educação, a serviços de saúde, a espaços de lazer, entre outros.

A estratégia de ensino pode ser considerada inovadora, porque estimula o estudante ao trabalho em grupo com diversos atores envolvidos na vigilância em saúde e ao debate sobre os conceitos de morbidade, letalidade, prevalência e incidência, tendo como ponto de partida a desigualdade territorial das necessidades em saúde com vistas à atuação individual e populacional. 
Tais estratégias pedagógicas visaram a questionar a realidade, construir questões sobre o fazer em saúde e interrogar o cotidiano em que atuamos, com ênfase no fortalecimento da rede de serviços públicos que compõem o sistema de saúde brasileiro e que são o eixo condutor da formação dos profissionais de saúde em nosso país.

\section{Referências Bibliográficas}

ARREAZA Antonio Luis Vicente \& MORAES José Cassio de. "Vigilância da Saúde: Fundamentos, Interfaces e Tendências". Ciência d Saúde Coletiva, vol. 15, n. 4, jul. 2010, pp. 2215-2228.
NUNES Everardo Duarte. "A doença como Processo Social". In: CANESQUI, Ana Maria (org.). Ciências Sociais e Saúde no Ensino Médico. São Paulo: Hucitec, 2000, pp. 217-229.

OLIVEIRA Cátia Martins de \& CRUZ Marly Marques. "Sistema de Vigilância em Saúde no Brasil: Avanços e Desafios". Saúde Debate, vol. 39, n. 104, jan./ mar. 2015, pp. 255-267.

WALDMAN, Eliseu Alves. "Vigilância como Prática de Saúde Pública". In: CAMPOS, Gastão Wagner de Sousa; MINAYO, Maria Cecília de Souza; AKERMAN, Marco; DRUMONDJÚNIOR, Marcos \& CARVALHO, Yara Maria de (orgs.). Tratado de Saúde Coletiva. São Paulo: Hucitec; Rio de Janeiro: Fiocruz; 2006, pp. 295-315.

Publicado em 12/06/2018. 